\title{
IDENTIDAD NUMÉRICA Y CORREFERENCIALIDAD
}

\author{
Agustín Arrieta URTIZBEREA \\ Departamento de Lógica y Filosofía de la Ciencia \\ Universidad del País Vasco \\ ylparura@sf.ehu.es
}

RESUMEN: Este trabajo constituye una defensa del carácter relacional de la identidad. Algunos filósofos han puesto en duda la identidad en cuanto que relación; Armstrong es uno de ellos. Una réplica a sus argumentos, además de a aquellos que consideran la identidad en términos de correferencialidad, nos lleva a la aceptación de la identidad como relación de razón. También se propone una interpretación de los enunciados de identidad que resulte compatible con los data acerca de la identidad. En esta tarea, son relevantes tanto la distinción entre especificación y descripción de un dominio como algunas ideas propuestas en Perry 2001.

Palabras Clave: relación de razón, John Perry, dominio

SUMMARY: This work argues in favor of the relational nature of identity. Some philosophers cast doubts on identity as relation, Armstrong being one of them. This reply to his arguments specifically, and to those who consider identity in terms of co-referentiality, proposes that identity is a relation of reason. An interpretation of identity-statements, compatible with data concerning identity itself, is also presented. For this task both the distinction between specification and description of a domain, and some ideas put forth in Perry 2001 are relevant.

KEY WORDS: relation of reason, John Perry, domain

En las primeras líneas de "Sobre sentido y referencia", Frege pone sobre el tapete todas aquellas cuestiones y tesis básicas que afectan a la identidad y los enunciados de identidad. Es difícil superar en concisión y precisión el planteamiento fregeano.

(i) ¿Es la identidad una relación?

(ii) ¿Es la identidad una relación entre objetos o entre nombres de objetos?

(iii) " $a=a " \mathrm{y}$ " $a=b$ " son enunciados de distinto valor cognoscitivo.

Frege asume que la primera pregunta tiene una respuesta afirmativa. Sin embargo, dicha respuesta no parece ser obvia, ya que, como se verá a continuación, hay autores que se muestran escépticos con respecto al carácter relacional de la identidad. En cualquier caso, sólo respondiendo afirmativamente a (i) cabe formular y preocuparse 
por (ii). En cuanto a la segunda pregunta, Frege defendió en distintos momentos diferentes respuestas para la misma. Por otro lado, el problema de la distinción entre el valor cognoscitivo de " $a=a$ " y de " $a=b$ " ha generado una línea de reflexión que se ha extendido a todos los rincones del lenguaje. Dicha distinción constituye, además, uno de los centros en torno a los cuales se ha enfocado el debate entre (neo)fregeanos y (neo)russellianos con respecto a la interpretación de los enunciados de identidad.

Mi objetivo principal en este trabajo es defender la tesis de que la identidad sí es una relación; en particular (y en terminología medieval), se trata de una relación de razón. Haré esto en la sección 2. En la sección 3 argumentaré que no es una relación de correferencialidad entre nombres de objetos. Finalmente, en la sección 4, mostraré que todo ello es compatible con el datum expresado en (iii).

\section{Delimitación de la terminología y aspectos básicos}

(1.1) Entiendo la identidad como identidad numérica. Aristóteles lo expresa en los siguientes términos:

En efecto, solemos dar la designación de idéntico, bien por el número, bien por la especie, bien por el género: son idénticas en número las cosas en que los nombres son múltiples, el objeto, en cambio, único. (Aristóteles 1982, I, 7)

Se llamó idéntico con la mayor propiedad a lo numéricamente uno. (Aristóteles 1982, VII, 1)

Por lo tanto, no me ocuparé en este trabajo de relaciones como las de igualdad (o identidad cualitativa), indiscernibilidad, duplicación, equivalencia, identidad parcial o semejanza.

Tampoco pretendo que se tome la afirmación aristotélica como una definición de la identidad, ya que la propia expresión "definición de la identidad" puede ser problemática. ${ }^{1}$

(1.2) Enunciados de identidad paradigmáticos son los siguientes: "Bernardo Atxaga es José Irazu", "este caballo es Luma", "yo soy Agustín",... En ocasiones, recurro a la expresión " $a=b$ " como representante de los enunciados de identidad.

Los enunciados de identidad son relacionales, al menos desde un punto de vista gramatical. Una expresión relacional ("es", "es el mismo $F$ que",...) y dos términos singulares son sus componentes.

${ }^{1}$ Véase McGinn 2001, cap. 1. 
Por "término singular" entiendo "término directamente referencial", cuya contribución a la proposición expresada por una proferencia que lo contiene es su referente, haciendo de dicha proposición una proposición singular. Ejemplos de términos directamente referenciales son los nombres propios y los indéxicos. Dejo de lado, pues, las descripciones definidas, para las cuales considero adecuado (al menos en su uso atributivo) un análisis russelliano. ${ }^{2}$

Para cualquier perspectiva, como la aquí asumida, que acepte la existencia de términos directamente referenciales, la explicación del datum expresado en (iii) constituye un problema serio (siempre que " $a$ " y " $b$ " sean términos directamente referenciales).

(1.3) Distingo entre enunciado y proferencia. Los enunciados son entidades-tipo, es decir, universales, mientras que una proferencia es una entidad particular que es un ejemplar de un enunciado. Las proferencias están localizadas espacio-temporalmente. Los enunciados tienen propiedades como la de tener un significado. Estas propiedades, en cierto sentido, son heredadas por sus ejemplares, esto es, por las proferencias. Además, un ejemplar en cuanto tal también tiene sus propiedades; por ejemplo, la propiedad de expresar una proposición. La proposición es lo que la proferencia dice o, si se prefiere, el contenido referencial u oficial de la proferencia. La distinción enunciado/proferencia es (semánticamente) pertinente siempre que el enunciado contenga algún componente de carácter indéxico. En el caso de los enunciados eternos, estas distinciones no tienen efectos (semánticos). Distinciones análogas se pueden plantear al nivel de los componentes de un enunciado. Por ejemplo, "yo" como entidad-tipo o universal tiene un significado, y además una proferencia de "yo" tiene o expresa un contenido. El contenido de la proferencia "yo", en caso de que Agustín sea el proferente, es el particular Agustín.

Si bien en algunas partes de este trabajo, y siguiendo en gran medida el uso habitual, recurriré a las expresiones "enunciado" y "enunciado de identidad", deberá entenderse, cuando el contexto así lo exija, que me estoy refiriendo a proferencias (de enunciados o de enunciados de identidad).

${ }^{2}$ Quedan fuera incluso las descripciones definidas rígidas, ya que un enunciado (o proferencia) que contiene una descripción rígida no expresa una proposición singular, es decir, no expresa una proposición que tiene como constituyente al referente de la descripción. Una descripción definida rígida es un designador rígido de facto, no de jure (Kripke 1980, nota 21). 


\section{2. ¿Es la identidad una relación?}

El problema de la existencia de las relaciones es un gran problema filosófico. Mi objetivo en esta sección no pasa por la resolución de tamaña cuestión. Voy a suponer que efectivamente hay relaciones, para tratar a continuación de contraatacar dos argumentos que han pretendido mostrar que la identidad no es una relación. "La gramática de la expresión de la identidad, por así decirlo, nos engaña una vez más, haciéndonos creer que lo que no es una relación sí lo es", afirmarían dichos autores. Un representante de este tipo de escepticismo es D.M. Armstrong. ${ }^{3}$

Armstrong se sitúa en una tradición de filósofos que han recelado de la relación de identidad. En esta tradición se encuentran algunos autores medievales, quienes motivados fundamentalmente por reflexiones acerca de la identidad (y de otras relaciones de marcado carácter teológico) sufrieron una evolución en su concepción de las relaciones. Reflexiones sobre la relación de identidad motivaron una evolución desde posiciones realistas hasta posiciones más nominalistas. No en vano la relación de identidad era calificada como relación de razón para contrastarla, precisamente, con las relaciones reales. ${ }^{4}$

En esta sección defenderé la idea de que en el caso de la identidad estamos ante una relación especial, sui generis, sin que por ello deje de ser una relación. Quizás el término "relación de razón" pueda ser útil para dar cuenta de estos aspectos.

¿En qué sentido es la identidad una relación?

Caben distintas respuestas. La identidad, por ejemplo, es una relación en el sentido de que es tratada como cualquier otra relación: se estudian sus propiedades (reflexividad, simetría, etc.) y se describen dichas propiedades mediante enunciados relacionales y cuantificados. Por ejemplo, decimos que la relación de identidad es una relación simétrica: $\forall x \forall y(x=y \rightarrow y=x)$. Si miramos las relaciones desde un punto de vista extensional, es decir, como conjuntos de pares ordenados, la identidad es la menor relación reflexiva que es posible definir en un dominio. Es así como podemos entender que la identidad es una relación. Sin embargo, podría alegarse que este concepto de relación es conjuntista y, por ende, generoso, ya que

\footnotetext{
${ }^{3}$ Véase Armstrong 1978. De otros tipos de escepticismo me he ocupado en Arrieta 1999 y 2001.

${ }^{4}$ Mi objetivo no es ocuparme de la concepción medieval sobre las relaciones, aunque, como se verá, sí haré uso de cierta terminología medieval. Todas mis consideraciones en esta sección acerca de la concepción medieval provienen de Brower 2001.
} 
partiendo de él se pueden sobregenerar relaciones o definirse relaciones irrelevantes desde un punto de vista ontológico. El problema es que en esta perspectiva conjuntista cabe una sobregeneración ontológica. Necesitamos, pues, un criterio ontológico que establezca qué relaciones hay. Esto es lo que nos interesa cuando discutimos sobre el carácter relacional de la identidad. Armstrong, por ejemplo, establece un criterio ontológico en términos de eficacia causal: sólo existen los universales (propiedades o relaciones) que dotan a los particulares que los instancian de poderes causales.

Abogaré por un criterio que no sea tan laxo como el conjuntista, ni tan estrecho como el causal. El primer criterio nos permite concluir que la identidad sí es una relación, mientras que el segundo nos lleva a la tesis opuesta. El criterio que propondré nos permite concluir, por razones distintas de las proporcionadas por el criterio conjuntista, que la identidad sí es una relación. Sin embargo, el hecho de que, de acuerdo con Armstrong, la identidad no dote de poderes causales a sus instanciadores nos lleva a concluir que la identidad es una relación de razón. Consideremos más detalladamente los argumentos de Armstrong "contra" la identidad.

El objetivo final de Armstrong es justificar que las únicas relaciones que hay son las relaciones espacio-temporales y las relaciones causales. En el desarrollo de su argumento, va eliminando posibles candidatos a tomar parte en ese conjunto de relaciones; una de las candidaturas rechazadas es precisamente la de la relación de identidad. Armstrong plantea fundamentalmente dos argumentos, uno epistemológico y otro ontológico. ${ }^{5}$ Con respecto al primero, Armstrong afirma que sabemos a priori que todo objeto es idéntico a sí mismo (también se sabe a priori que todo objeto se parece a sí mismo, o que tiene el mismo tamaño que sí mismo). Para Armstrong, esto prueba que la supuesta relación de identidad no es una relación, aunque poseamos una expresión relacional de la identidad. Armstrong asume como principio que sólo puede determinarse $a$ posteriori —mediante la investigación empírica - cuáles son las relaciones (y los universales, en general). ${ }^{6}$ El segundo argumento, por su parte, niega el carácter relacional de la identidad en virtud de su ineficacia causal: la supuesta relación de identidad (o la propiedad

${ }^{5}$ Armstrong 1978, pp. 10-11 y 91-94. En las pp. 10-11, el objetivo es la relación de identidad, mientras que en las pp. 91-94, los mismos argumentos se generalizan a toda relación reflexiva.

${ }^{6}$ Armstrong 1978, p. 11. 
de ser idéntico a sí mismo) no dota al objeto relacionado (consigo mismo) de ningún tipo de poder causal. ${ }^{7}$

¿Cuál es el problema con el argumento epistemológico? Con respecto a cualquier objeto sabemos a priori que ese objeto es idéntico a sí mismo. En opinión de Armstrong, ese hecho es suficiente para rechazar la identidad como relación. ${ }^{8}$ La razón esgrimida es que el conocimiento a priori de la reflexividad de la identidad va en contra del principio antes señalado: sólo la investigación empírica puede determinar cuáles son las relaciones.

Este argumento dista mucho de ser claro y, por lo tanto, concluyente. Efectivamente, podemos aceptar que la determinación de cuáles son las propiedades y las relaciones es algo que compete a la investigación científico-empírica (dejaremos a un lado la matemática). Siendo esto así, la determinación de cuáles son las propiedades y las relaciones pasa también por un análisis de las teorías científico-empíricas, teorías en las que se expresa el conocimiento científicoempírico. Entiendo que una teoría científica es un conjunto (con determinadas características) de enunciados. La pregunta pertinente en este punto es la siguiente: ¿qué papel desempeñan los enunciados de identidad en dichas teorías? Quizás no esté reñido el hecho de que un enunciado de identidad forme parte de una teoría empírica con el hecho de que la identidad sea ( $a$ priori) reflexiva.

Saber a priori que $p$ no implica saber a priori que un enunciado (una proferencia) que expresa $p$ sea verdadero. Por ejemplo, no sabemos a priori que "Héspero es Fósforo" expresa una proposición verdadera, aunque sabemos a priori que Venus es Venus. Lo relevante es que el enunciado "Héspero es Fósforo" desempeña un papel en nuestra teoría científico-empírica, ya que sabemos a posteriori que dicho enunciado expresa una proposición verdadera (y a priori).

La relación conversa tampoco parece darse. Podemos saber a priori que un enunciado expresa una proposición verdadera, sin que ello implique que sepamos a priori que $p$ (la proposición expresada) es verdadera. Cualquier hablante competente del castellano sabe $a$ priori que una proferencia de "yo estoy aquí ahora" expresa una proposición verdadera, pero de ahí no se deriva que sepa a priori la proposición expresada. Si enumeramos las proferencias de ese enunciado hechas en un día $d$, el hablante sabe que la proferencia

${ }^{7}$ En las páginas 10-11, Armstrong (1978) se ocupa de la propiedad (relacional) de ser idéntico a sí mismo; sin embargo, en las páginas 91-94, aplica los mismos argumentos a la relación de identidad (y a otras relaciones reflexivas).

${ }^{8}$ Armstrong 1978, pp. 11 y 92. 
número 5 expresa una proposición verdadera, pero no sabiendo, por ejemplo, quién realizó dicha proferencia, no conocerá la proposición expresada y, por ende, no podemos decir que sabe a priori dicha proposición. $^{9}$

Lo importante es que una teoría es un conjunto de enunciados que se incorporan a la misma a partir de investigaciones empíricas, aun cuando esos enunciados expresen proposiciones de las cuales se sabe a priori que son verdaderas. En este sentido, la incorporación de un enunciado de identidad a una teoría científico-empírica sí es fruto de una investigación empírica y este hecho es independiente del carácter a priori de la proposición expresada por dicho enunciado.

Considero que la búsqueda de una razón para rechazar la identidad como relación debe orientarse en otra dirección; debe vincularse con algún criterio ontológico. El segundo argumento propuesto por Armstrong señala claramente cuál es, en su caso, ese criterio.

Antes de ocuparme de dicho argumento, vale la pena decir algo más acerca de por qué el debate de fondo es ontológico. Veamos un argumento de Quine. Éste escribe: "Lo que hace de la identidad una relación, y de ' $=$ ' un término relacional, es que ' $=$ ' aparece entre ocurrencias distintas de términos singulares (del mismo o de distintos), y no el hecho de que relacione objetos distintos" (Quine 1960, p. 116). ${ }^{10}$

Efectivamente, los enunciados de identidad "interesantes" en las teorías científico-empíricas tienen la forma " $a=b$ ", donde " $a$ " y " $b$ ", como Quine señala, son ocurrencias distintas del mismo o de distintos términos singulares. A partir de esta característica Quine concluye que la identidad sí es una relación, a pesar de su reflexividad. Este (supuesto) argumento a favor de la naturaleza relacional de la identidad tampoco resulta satisfactorio. Cualquier enunciado relacional " $a R b$ " cumple esa condición, y a partir de ese hecho no cabe concluir que cualquier expresión gramaticalmente relacional exprese una relación. La razón de fondo para concluir aquí que la identidad sí es una relación debe estar vinculada, al igual que en el caso de Armstrong, con algún criterio ontológico. Luego, por lo que se ha visto con respecto a los argumentos de Armstrong y Quine, la reflexividad (a priori) de la identidad no permite tomar decisiones acerca de la

${ }^{9}$ Este ejemplo, propuesto por un árbitro anónimo, mejora el que yo propuse en una versión previa de este trabajo.

${ }^{10} \mathrm{~A}$ menos que se indique de otra manera, la traducción de los textos citados es mía. 
naturaleza relacional de la identidad. El debate de fondo es más bien ontológico.

El argumento ontológico de Armstrong para extraer la identidad del reino de lo que hay se basa en su ineficacia causal (expresada en los términos antes señalados). En la terminología medieval, si acaso este argumento muestra algo, muestra que la identidad no es una relación real, pero no muestra que no sea una relación. Como se verá, la identidad es una relación de razón.

En la perspectiva de Armstrong, los componentes básicos de la ontología (particulares y universales) están "encadenados" de alguna forma a la relación causal: "Si entre las relaciones (externas) con las que realmente estamos familiarizados hay alguna que sea fundamental desde un punto de vista ontológico, ésa, sospecho, es la causalidad. Quizás sea el cemento del universo" (Armstrong 1997, p. 89). ¿Qué ocurre con las otras relaciones? ¿Qué ocurre con la identidad numérica? La identidad es una relación interna. También lo son, por ejemplo, las relaciones de diferencia, semejanza y contrariedad. Las relaciones externas son las relaciones que no son internas. La relación externa fundamental es, como se ha dicho, la relación de causalidad. En cualquier caso, lo importante es que, refiriéndose a las relaciones externas, Armstrong afirma: "Las relaciones externas son aquellas que no son internas, y son las relaciones importantes desde un punto de vista ontológico" (Armstrong 1997, p. 87). Contrariamente, las relaciones internas no son nada más allá de los elementos supuestamente relacionados: "[L]as relaciones internas no añaden nada al mobiliario del mundo" (Armstrong 1997, p. 87). La razón es que las relaciones internas no aportan nada desde el punto de vista causal. No es mi objetivo evaluar las tesis asumidas en este tipo de perspectiva, aunque retomaremos el tema a continuación, al exponer brevemente las ideas de Wittgenstein. Es obvia la sintonía entre las tesis que ambos autores defienden con respecto a la identidad; sin embargo, parece que en las teorías empíricas recurrimos a enunciados relacionales de identidad que no pueden ni eliminarse ni reducirse a otros enunciados o expresiones. Hay, pues, cierta tensión entre lo que las teorías indican y lo que el criterio de Armstrong establece.

Quine propone un criterio ontológico distinto del de Armstrong: una teoría está obligada a admitir aquellas entidades a las cuales tienen que referirse las variables ligadas de la teoría para que las afirmaciones hechas en ésta sean verdaderas (Quine 1948). La teoría se ocupa de un dominio de objetos. Olvidémonos por un momento de 
"prejuicios quineanos" con respecto a los universales y formulemos el siguiente principio (cuasi)quineano: una teoría está obligada a admitir aquellas entidades a las cuales tienen que referirse las variables ligadas, los nombres propios, los predicados y las expresiones relacionales de la teoría para que las afirmaciones hechas en ésta sean verdaderas. Mediante la teoría queremos afirmar cuáles son las propiedades-de y las relaciones-entre los objetos en el dominio. Estas propiedades y relaciones son expresadas por predicados y expresiones relacionales respectivamente. Entre los elementos de la teoría tenemos prima facie los enunciados de identidad. En principio hay una expresión relacional en la teoría que expresa la identidad. Por lo tanto, según el criterio (cuasi)quineano, la identidad sí es una relación. En cualquier caso, cabe la siguiente réplica. Si un enunciado de identidad es verdadero, afirma de un objeto que es idéntico a sí mismo. Por otro lado, y de acuerdo con Armstrong, la identidad no es causalmente pertinente. Además, el enunciado " $a=b$ " no proporciona directamente información descriptiva alguna acerca del objeto $a$. En consecuencia, cabe pensar que podemos "prescindir" en dicha teoría del enunciado de identidad, y bloquear de esa forma el argumento que nos llevaba a aceptar la identidad como relación. En ocasiones, se han propuesto estrategias eliminacionistas y reduccionistas con respecto a las relaciones en general, y con respecto a la identidad en particular. Por ejemplo, en la filosofía medieval se argumentaba que tras toda expresión relacional " $a R b$ " se encuentran dos propiedades (accidentales) $P$ y $Q$ tales que $P a$ y $Q b$, por así decirlo, son hacedores de verdad de " $a R b$ ". Los medievales, en general, rechazaron la existencia de propiedades poliádicas. En el caso de la identidad, ese tipo de reducción de las relaciones a otros universales no poliádicos, en caso de ser factible, resultaría extremo, ya que a un enunciado de identidad " $a=b$ " correspondería, en la ontología, el objeto $a$ (o $b$ ) sin más. Con esto, la identidad entre $a$ y $b$ se reduciría, ontológicamente hablando, a $a$ (o a $b)$ y en la teoría prescindiríamos del enunciado de identidad: basta con un nombre para dicho objeto. Wittgenstein, otro escéptico con respecto a la identidad, hace ese tipo de planteamiento. Su argumento en contra del carácter relacional de la identidad es que en el lenguaje lógico la función atribuida al símbolo de identidad puede ser efectuada por el nombre del objeto. En el lenguaje lógico, los enunciados de identidad sobran; basta con un nombre para cada objeto. ${ }^{11}$ No es mi objetivo profundizar en esta estrategia eliminacionista wittgensteiniana, pero parece claro que trae

${ }^{11}$ Wittgenstein 1921, 5.53, 5.5301, 5.533. La reducción que proclama Wittgen- 
consigo una concepción correctiva, que no descriptiva, de nuestra actividad lingüística y epistémica. Renunciar al símbolo de identidad sería ocultar toda la actividad epistémica que gira en torno a los enunciados de identidad: fundamentalmente, "descubrimientos" de identidades y diferencias. En este trabajo asumo que los enunciados de identidad están presentes en nuestras teorías de forma ineliminable e irreducible. ${ }^{12}$

Anteriormente he señalado que los enunciados de identidad no aportan información descriptiva acerca del objeto en cuestión. Entonces, ¿cuál es su función? El descubrimiento de la verdad de un enunciado de identidad tiene efectos sobre nuestra concepción de la ontología; en particular, tiene efectos sobre nuestra especificación del dominio de la ontología que investiga la teoría, e indirectamente o inferencialmente sobre la descripción de los objetos en dicho dominio. La especificación del dominio no es transparente, es decir, no podemos afirmar a priori acerca de los términos singulares utilizados para referirnos a los elementos del dominio si se refieren a un mismo elemento o no. Los enunciados de identidad cumplen una función a este respecto. ${ }^{13}$

Podemos resumir lo señalado hasta el momento en los siguientes términos. No toda expresión relacional correctamente definida desde un punto de vista conjuntista expresa una relación. En caso contrario, se produciría una sobregeneración ontológica. Tampoco cabe aplicar un criterio ontológico basado en principios ontológicos independien-

stein es estructuralmente análoga a la propuesta por Armstrong; sin embargo, los argumentos de ambos a favor de la misma son distintos.

${ }^{12}$ Quine propone un análisis de la identidad de $a$ y $b$ en términos de indistinguibilidad con respecto a los predicados y, por ende, con respecto a las expresiones predicativas (complejas) del lenguaje (Quine 1970, cap. 5). A partir de esa idea podemos eliminar la expresión de identidad a favor de una expresión compleja que abarca todas las combinaciones al caso que puedan generarse en el lenguaje. El éxito del proyecto asume que el número de expresiones predicativas sea finito. De cualquier modo, hay que distinguir identidad y sustituibilidad salva veritate. El segundo concepto, a diferencia del primero, es dependiente del poder expresivo del lenguaje. Por ello es posible la sustituibilidad sin identidad.

El método quineano busca la eliminación, pero caben estrategias más débiles que pretenden la reducción de la identidad a (o la superveniencia de la identidad sobre) otras propiedades y relaciones (que no presupongan la identidad). Estoy de acuerdo con Wiggins (2002) en lo que se refiere a las dificultades de ambos proyectos. La aceptación de la eliminabilidad, reducibilidad o superveniencia de la identidad constituye otro tipo de escepticismo con respecto a la misma. No me ocuparé aquí de esta cuestión.

${ }^{13}$ Sobre la distinción entre especificación y descripción de un dominio, véase Arrieta 2001. En la sección 4 retomaré con más detalle estos aspectos. 
tes de cualquier consideración relacionada con nuestras teorías. Las teorías señalan qué relaciones hay. Según el criterio (cuasi)quineano y sobre la base de las consideraciones desarrolladas anteriormente, debemos concluir que la identidad sí es una relación; sin embargo, habiendo asumido que la identidad es causalmente ineficaz, diremos que no se trata de una relación real, sino más bien de una relación de razón.

\section{3. ¿Es la identidad una relación entre nombres de objetos?}

Es conocida la historia. Frege en la Conceptografía (parágrafo 8) defiende una salida ad hoc para la identidad. La identidad sí es una relación, pero es una relación entre signos, entre nombres de objetos. En el mismo lugar, Frege dice que a diferencia del juntor condicional que relaciona contenidos, la identidad relaciona nombres, por lo cual una vez que introducimos en el lenguaje el signo de identidad, todos los signos adquieren un doble significado: un símbolo a veces se representa a sí mismo, y a veces representa otra cosa. El nombre "Héspero" en ocasiones se significa a sí mismo y, en otras ocasiones, lo usamos para hablar de un planeta determinado: se refiere a sí mismo en "Héspero = Fósforo" y se refiere a un planeta en "Héspero aparece al atardecer". La inclusión del símbolo de identidad, pues, tiene un efecto traumático para el lenguaje.

Frege da respuesta de esta forma a nuestras dos primeras preguntas: la identidad es una relación y relaciona nombres de objetos. Con respecto al datum fregeano (expresado en (iii)), la respuesta de Frege es la siguiente: los enunciados " $a=b$ " y " $a=a$ " tienen distinto valor cognoscitivo ya que en el caso de " $a=b$ " se expresa una relación de mismidad de contenido (o de mismidad de referencia) de dos signos distintos, mientras que " $a=a$ " expresa la mismidad de contenido de un signo consigo mismo (o de dos instancias de un mismo signo). Esta propuesta de Frege puede tomarse como un antecedente de una propuesta correferencialista de la identidad. Así, los enunciados de identidad expresan una relación de correferencialidad.

Sin embargo, el que los signos a la izquierda y a la derecha de la expresión de identidad sean distintos no es una condición ni necesaria ni suficiente para dar cuenta de la diferencia con respecto al valor cognoscitivo. Hay enunciados de identidad a posteriori que tienen a la izquierda y a la derecha el mismo nombre, refiriéndose a lo mismo. Piénsese en el caso Paderewski. ${ }^{14}$ Una persona conoce a

\footnotetext{
${ }^{14}$ Kripke 1979. Para comentarios sobre este caso, véase Perry 2001, pp. 113-116.
} 
Paderewski, un excelente pianista. La misma persona sabe que hay un político llamado "Paderewski". Un día descubre que se trata de la misma persona, es decir, descubre a posteriori que Paderewski es Paderewski. Por otro lado, hay casos en los que las expresiones a la izquierda y a la derecha del signo de identidad son distintas $\mathrm{y}$, no obstante, el enunciado de identidad es a priori. Piénsese en una proferencia de "este varón no casado es este soltero" (Noonan 2001). Parece, pues, que esa forma de interpretar los enunciados de identidad no está exenta de problemas.

Si bien esto podría verse como un problema posible que afecta a la teoría propuesta en la Conceptografía, no parece que Frege lo tuviera in mente. A pesar de ello, Frege evoluciona y en "Sobre sentido y referencia" pasa a defender que la identidad es una relación entre objetos. He aquí un argumento que justificaría esa evolución: a consecuencia de la primera concepción, una vez introducido el símbolo de identidad en un lenguaje, todos los símbolos adquirirían un significado doble, lo cual parece ir en contra de los hechos. Cuando escribió "Sobre sentido y referencia", Frege era consciente de la contundencia de este argumento en contra de las ideas que defendió en la Conceptografía. De hecho, en otro contexto, es el propio Frege quien hace uso de un argumento de estructura similar. En "Función y concepto", Frege critica las "teorías formalistas de la aritmética", según las cuales las expresiones numéricas se refieren a las expresiones mismas. En dicho texto, Frege esgrime un argumento que podría resumirse de la siguiente forma: si las expresiones numéricas se refirieran a ellas mismas, entonces las expresiones complejas en las que aparecen expresiones numéricas se deberían entender también de una manera distinta de la habitual. Por ejemplo, "tiene la propiedad de" significaría cosas distintas en "Juan tiene la propiedad de ser barbudo" y en "3 tiene la propiedad de ser impar", ya que, en el primer caso, "Juan" no se refiere a la expresión "Juan", mientras que, según el formalista, "3" sí se refiere a la expresión "3". En consecuencia, en el primer caso afirmamos que una persona tiene una propiedad. ¿Qué afirmamos en el segundo caso? Si "3" se refiere a una expresión, entonces, manteniendo el significado de "tiene la propiedad de", estaríamos afirmando que dicha expresión tiene la propiedad de ser impar, lo cual parece absurdo: "Pero tal concepción no puede mantenerse, porque no podemos hablar en absoluto de cualesquiera propiedades aritméticas de los números, sin referirnos a la referencia de los signos numéricos" (Frege 1891, p. 149; las cursivas son mías). 
Cabe pensar que "tiene la propiedad de" tiene un significado diferente en esos dos enunciados. En este caso, quizás plausible, estaríamos creando un problema de doble significado que va más allá de las expresiones numéricas.

Frege es consciente de las razones que el formalista tiene a su favor. Básicamente hay una: el referente de un signo numérico, según la teoría no formalista, es una entidad "extraña", en el sentido de que es una entidad no sensiblemente perceptible. La salida del formalista para evitar el problema de la aceptación de entidades que no son sensiblemente perceptibles es, según Frege, crear un problema mayor.

El caso de la identidad resulta paralelo. Hay un problema (" $a=a$ " y " $a=b$ " tienen diferente valor cognoscitivo) y para solucionarlo se propone (Frege lo hace en la Conceptografía) una interpretación ad hoc de los enunciados de identidad; sin embargo, esa misma solución se convierte en un problema más serio que se extiende por todo el lenguaje. Si alguien afirma "Héspero aparece todas las tardes y además dicho planeta es Fósforo", parece que en todo momento está hablando acerca de un mismo planeta. Sin embargo, esa interpretación natural es problemática desde la concepción para la identidad propuesta en la Conceptografía. "Dicho planeta" no se refiere a Héspero sino a "dicho planeta", por lo que el doble significado comienza a expandirse a expresiones que no son nombres propios. Si entre los términos singulares que aparecen en el enunciado de identidad se consideran expresiones deícticas o anafóricas ("ese planeta", "dicho planeta", etc.) el problema se manifiesta claramente. Es posible extender el problema todavía más: ¿cómo interpretamos en el enunciado "Héspero aparece todas las tardes y además dicho planeta es Fósforo" la expresión "y además”?

Aunado a este problema, que considero que es el gran problema, pueden plantearse más argumentos en contra de la perspectiva correferencialista acerca de la identidad. Los siguientes argumentos se deben a Kripke.

(1) Supongamos que " $a=b$ " significa " $a$ ' y ' $b$ ' refieren a lo mismo". Si aceptamos que los enunciados de identidad verdaderos son necesariamente verdaderos, entonces tenemos que aceptar que el enunciado " " $a$ ' y ' $b$ ' refieren a lo mismo" también es necesariamente verdadero, si " $a=b$ " es verdadero. Esta última afirmación parece claramente falsa, ya que parece difícilmente defendible que "Héspero" y "Fósforo" se refieran necesariamente a lo mismo, es decir, se refieran a lo mismo en todo mundo posible; todo ello a pesar de que 
Héspero es Fósforo necesariamente. Cabe concebir un mundo posible en el que el nombre "Héspero" se refiera a un planeta $c$ en virtud de una convención, mientras que "Fósforo" se refiere a una estrella $e$ en virtud de otra convención: todo ello mientras Héspero sigue siendo Fósforo en ese mismo mundo. $\mathrm{O}$ cabe concebir un mundo en el que simplemente no haya lenguaje y en el que, en consecuencia, no tendría sentido hablar de la correferencialidad, mientras que Héspero seguiría siendo el mismo planeta que Fósforo. Luego, " $a=b$ " y " " $a$ ' y ' $b$ ' refieren a lo mismo" no son ni siquiera necesariamente coextensionales. Por lo tanto, tampoco tienen el mismo significado. Una debilidad de este argumento es que asume la necesidad de la identidad, ${ }^{15}$ por lo que cualquier duda sobre esta tesis lo ensombrecerá. ${ }^{16}$

(2) Kripke hace algunos comentarios breves sobre la concepción correferencialista de la identidad. Aun si este concepto de identidad es aceptable, se puede introducir una nueva relación mediante la denominación "schmidentidad": se trata de la relación que cada objeto tiene sólo consigo mismo. ${ }^{17} \mathrm{Al}$ entender la identidad como correferencialidad, parece ser que resolvemos los problemas de la identidad (en particular, el problema relacionado con el datum expresado en (iii)). Pero ahora el mismo problema se plantearía con respecto a " $a$ es schmidéntico a $b$ ". Lo que Kripke parece estar diciendo es que entender la identidad como correferencialidad no va a librarnos de los enigmas y tesis acerca de la menor relación reflexiva.

Ese argumento supone un concepto de relación lejano al propuesto por Armstrong y compatible con la concepción que he descrito antes (Secc. 2). No en vano el propio Kripke señala que hay filósofos que consideran que una relación es esencialmente diádica (o, podemos añadir, poliádica), por lo que para esos filósofos no tiene sentido hablar de una relación que relaciona un objeto consigo mismo. ${ }^{18}$ Kripke califica de absurda esa concepción de las relaciones, y aunque no especifica a qué filósofos se está refiriendo, en dicha descripción encajaría perfectamente Armstrong, quien en la afirmación que cito a continuación descarta (aunque sólo tentativamente) las relaciones reflexivas: "Tentativamente, sugiero que se debería aceptar un principio de invarianza con respecto a la instanciación: para todo $n$, si

${ }^{15}$ Kripke 1971, p. 142.

${ }^{16}$ Por ejemplo, en Gallois 1998 se dan argumentos contra la tesis de la necesidad de la identidad.

${ }^{17}$ Kripke 1980, inicio de la tercera conferencia.

${ }^{18}$ Kripke 1980, p. 108, n. 50. 
un universal es $n$-ádico con respecto a una instanciación particular, entonces es $n$-ádico con respecto a todas sus instanciaciones" (Armstrong 1978, p. 94).

Luego, lo que para Kripke es absurdo para Armstrong es aceptable.

En cualquier caso, como se ha señalado, el argumento propuesto por el propio Frege (en otro contexto) constituye el desafío más serio a la identidad entendida como correferencialidad.

\section{La identidad como relación entre objetos.}

Hacia una interpretación de los enunciados de identidad

La identidad es una relación sui generis. La identidad es una relación cuyas propiedades son conocidas a priori: es reflexiva y cumple la ley de Leibniz. El hecho de que sea una relación cuyas propiedades se conocen a priori no la hace especial, pero sí el hecho de que sea la única relación que cumple esas dos propiedades: dado un dominio es la menor relación reflexiva definida en ese dominio. Directa o indirectamente, este hecho ha desencadenado sospechas sobre la misma; sin embargo, no se sabe a priori si un enunciado de identidad expresa una proposición verdadera. En general, el enunciado " $a=b$ ", que expresa que el objeto $a$ (o $b$ ) es idéntico a sí mismo, no es inofensivo epistémicamente hablando, a diferencia del enunciado " $a=a$ ". ${ }^{19}$ Además, los enunciados de identidad forman parte del entramado de nuestras creencias acerca del mundo en igualdad de condiciones con otros enunciados, sin generar traumas en el interior del lenguaje mediante el que expresamos dichas creencias. Éstos son los data a los que una teoría de la identidad se debe enfrentar.

Frege en "Sobre sentido y referencia" encuentra la llave que le permite explicar toda la relevancia epistémica asociada a los enunciados de identidad; se trata del concepto de sentido. Pero surge un problema, y la bibliografía que ha emanado de "Sobre sentido y referencia" da prueba de ello: el concepto de sentido dista mucho de ser claro y distinto. Frege ha visto con claridad que una teoría de dos únicos componentes (el nombre y el contenido semántico en tiempos de la Conceptografía) no puede dar cuenta de los data. Su conclusión es que hace falta un tercero; pero los textos fregeanos han dado lugar a diferentes interpretaciones acerca del tercer componente propuesto. El sentido no es el significado ni tampoco es una representación

\footnotetext{
${ }^{19}$ Digo "en general", teniendo presente la discusión de la sección 3 sobre "Paderewski es Paderewski" y "este varón no casado es este soltero".
} 
(psicológica), aunque sí es objetivo (o intersubjetivo), determina el valor cognoscitivo y la referencia, y constituye un modo de presentación de la referencia, la cual, a su vez, no es un componente del sentido. Además, el sentido es aquello que captamos al aprender a usar una expresión. No está claro que todas estas características sean compatibles; y sí es discutible que el fregeano deba comprometerse con todas ellas. Quizás se pueda ser fregeano postulando el sentido como componente semántico sin caer en un platonismo en el que se acepta que la referencia no forma parte del sentido. ${ }^{20}$ En cualquier caso, el fregeano asume que el contenido semántico asociado con una expresión token se desglosa en dos componentes: el sentido y la referencia.

En una serie de trabajos recientes, John Perry ha retomado el problema de la identidad al hilo del planteamiento fregeano. Perry propone un marco teórico que trata de explicar, en primer lugar, el fenómeno de la indexicalidad. Quizá ésta fue para Frege, como para otros lógicos clásicos, un fenómeno marginal en el interior del lenguaje. En la actualidad, esta perspectiva fregeana se ha puesto en entredicho. Perry ha hecho su propuesta acerca del lenguaje tomando como punto de partida un fenómeno extendido a lo largo de todo el lenguaje como es el de la indexicalidad (y más precisamente, el fenómeno de la reflexividad que, en el caso de los indéxicos es manifiesto). Su propuesta es no fregeana, ya que el contenido semántico de la proferencia de un término singular es exclusivamente su referencia y el contenido semántico (también llamado "contenido oficial" o "contenido referencial") de la proferencia de un enunciado singular es una proposición singular. Es sabido que toda propuesta no fregeana del contenido semántico debe enfrentarse a dos problemas, a saber, el problema de la interpretación de los enunciados singulares de (no) existencia y de los enunciados de identidad.

La idea básica de Perry es que para dar cuenta del problema relacionado con la diferencia de valor cognoscitivo entre " $a=a$ " y " $a=b$ ", no basta con el contenido referencial. Perry recurre al concepto de contenido no referencial (no oficial). En la especificación del contenido no referencial hay elementos claramente reconocibles: el significado de las expresiones-tipo, el contexto de proferencia y la proferencia misma, nuestro conocimiento de todos ellos, las convenciones lingüísticas que se consideran en dicho contexto, el conocimiento compartido por los interlocutores, etc. Este contenido no referencial, además, se desglosa en distintos niveles.

${ }^{20}$ García Carpintero 1996, caps. VII y VIII. 
Consideremos un ejemplo. Supongamos que estoy en una estación de tren y me dirijo a Madrid por primera vez. Supongamos que escucho una proferencia de "yo estuve ayer en Madrid". El contenido referencial u oficial de dicha proferencia sólo me es accesible si se dan una serie de condiciones que afectan a distintos tipos de conocimiento. Supongamos que el contenido oficial es que Juan Valdés estuvo en Madrid el día 11 de junio de 2003. Para acceder a eso que ha dicho el proferente —al contenido oficial - tengo que saber castellano y, en consecuencia, tengo que conocer el significado de "yo", tengo que saber que el día de la proferencia es el 12 de junio, tengo que saber que el proferente es Juan Valdés, tengo que saber que Madrid es la ciudad asociada con el nombre "Madrid" a través de una convención que está siendo considerada en dicha proferencia, etc. Si, por ejemplo, esa proferencia la he escuchado estando en un lugar desde el que no veo al proferente, y además no tengo la menor idea de quién pueda ser, entonces tendré acceso a un contenido asociado con dicha proferencia que no es el contenido oficial de la misma. Dicho contenido tendría esta forma: el proferente de dicha proferencia estuvo en Madrid el día 11 de junio. Si no sé que día es hoy, entonces el contenido aprehendido es: el proferente de dicha proferencia estuvo en Madrid el día anterior al de la emisión de dicha proferencia. Éste es un contenido, según Perry, reflexivo ya que en él se hace referencia a la proferencia misma. En cualquier caso, y dejando los detalles a un lado, la estrategia de Perry es asociar con una misma proferencia una escala de contenidos. En el límite está el contenido referencial u oficial. Pero, además de éste, existe toda una escala de contenidos que se determinan en función de nuestro conocimiento lingüístico y no lingüístico en torno a la expresión proferida. Si para dar cuenta de los problemas cognoscitivos concernientes a la identidad, Frege recurría al concepto de sentido, Perry lo que hace es establecer este concepto gradual de contenido no referencial.

El sentido fregeano, en el caso de la proferencia de un enunciado, "determina" tanto su valor cognoscitivo como su valor veritativo (referencia). Lo "determina" ya que, por un lado, proferencias con distinto valor cognoscitivo no pueden tener el mismo sentido y, por otro lado, proferencias con distinto valor veritativo tampoco pueden tener el mismo sentido. En definitiva, el sentido "cuida" tanto del valor cognoscitivo como del veritativo. Perry, sin embargo, considera que es necesaria una ruptura dentro del sentido fregeano: hay que distinguir dos componentes, cada uno de los cuales lleva a cabo una de las dos funciones atribuidas al sentido fregeano. El primer com- 
ponente "dirige la mirada" hacia el valor veritativo de la proferencia. Se trata del contenido referencial u oficial de dicha proferencia. Se trata, en definitiva, de la proposición expresada por la proferencia. Éste es el contenido semántico oficial de la proferencia. En lo que respecta al valor cognoscitivo, los contenidos no referenciales adquieren protagonismo. Frege pretende dar cuenta de todo ello mediante el sentido; sin embargo, y expresándolo de forma poco rigurosa, en la perspectiva fregeana se le exige demasiado al sentido. Cuando la proferencia de un enunciado tiene como componente la proferencia de una expresión indéxica, la tensión interna al concepto de sentido se hace manifiesta. ${ }^{21}$

Retomando el problema que nos ocupa, la teoría de Perry es superior a la fregeana en cuanto a poder explicativo se refiere, por la sencilla razón de que los enunciados de identidad que consideramos son de la forma " $a=b$ ", donde " $a$ " y " $b$ " son términos singulares, y entre estos términos singulares se encuentran expresiones indéxicas para las que Frege no parece dar un tratamiento satisfactorio. Una razón que puede explicar este hecho es que Frege aterriza en la cuestión de los indéxicos tras haber desarrollado el concepto de sentido principalmente con respecto a los nombres propios, probablemente porque para Frege la indexicalidad es un fenómeno de poca relevancia. Sin embargo, la indexicalidad es un fenómeno mucho más

${ }^{21}$ En Perry 1977 se expone esa tensión en el interior del concepto de sentido. Es posible hacer una primera aproximación a dicha tensión mediante un ejemplo (con ello no se pretende decir la última palabra sobre el asunto). Sean $p_{1}$ y $p_{2}$ dos proferencias de "hoy está nevando". Supongamos que la primera proferencia es verdadera ya que el 3 de enero está nevando; mientras que la segunda es falsa ya que el 5 de enero no está nevando. En consecuencia, según el criterio que dice que proferencias con distinto valor veritativo tienen distinto sentido, $p_{1}$ y $p_{2}$ tienen sentidos distintos y, por lo tanto, "hoy" tiene sentidos distintos en las dos proferencias. Esta conclusión resulta chocante si consideramos, con Frege, que el sentido es aquello que captamos para aprender a usar una expresión. Es decir, parece que "hoy" tendría el mismo sentido en ambas proferencias (ese sentido podría ser, por ejemplo, el carácter kaplaniano), en contra del criterio.

Una solución (relativa) es pensar que el sentido de "hoy" en $p_{1}$ es el 3 de enero y en $p_{2}$ el 5 de enero (en general, el sentido de un indéxico vendría determinado por alguna descripción definida). De esta forma, ambas proferencias tendrían sentidos distintos, y el criterio antes mencionado quedaría intacto. En ese caso, la proferencia $p_{1}$ y una proferencia de "el 3 de enero está nevando" tendrían el mismo sentido; sin embargo, parece claro que $p_{1}$ y "el 3 de enero está nevando" tienen distinto valor cognoscitivo: una persona puede asentir ante $p_{1}$ y no hacerlo ante "el 3 de enero está nevando". Por lo tanto, según el otro criterio fregeano (proferencias con distinto valor cognoscitivo tienen distinto sentido), dichas proferencias tendrían distinto sentido, en contra de lo que se ha supuesto. 
importante y extendido de lo que Frege parece suponer. El planteamiento de Perry es el inverso: el punto de partida es el fenómeno de la indexicalidad (o, en general, el fenómeno de la reflexividad). Perry, pues, en lo que a la identidad se refiere, considera los data en el contexto de un marco más general, ya que los problemas de la identidad no sólo surgen en relación con proferencias de enunciados como "Héspero es Fósforo", sino también con relación a proferencias de "éste es éste" o de "yo soy Perry". Los mencionados contenidos no oficiales tienen la capacidad explicativa suficiente para abordar de una manera uniforme cuestiones relacionadas con el valor cognoscitivo de todas esas proferencias. Cabe añadir que los constituyentes de los contenidos perryanos son identificables y tienen cierto carácter manifiesto, además de vincularse directamente con los aspectos epistémicos que son tan relevantes entre los data en torno a la identidad.

Volvamos al ejemplo de la proferencia en la estación del tren ("yo estuve ayer en Madrid"). El contenido no referencial aprehendido permite explicar mi comportamiento, lo cual muestra la incidencia de dicho contenido en mis estados mentales. Suponiendo que yo nunca he estado en Madrid y que tengo la intención de informarme acerca de esa ciudad, dicha proferencia y la aprehensión del contenido reflexivo antes descrito (a pesar de no haber aprehendido su contenido semántico oficial) pueden ser suficientes para que me lance a la búsqueda del proferente con la intención de recabar de él información sobre Madrid. Si hubiera oído la proferencia "Juan Valdés estuvo en Madrid el día 11 de junio del 2003", mi comportamiento habría sido distinto, a pesar de que dicha proferencia y la proferencia "yo estuve ayer en Madrid" tienen el mismo contenido referencial.

¿Cómo interpretar en esta perspectiva la cuestión de la identidad y de su carácter relacional?

Lo que es dicho [mediante un enunciado] es lo que es dicho acerca del asunto que nos ocupe; se trata de cómo tiene que ser el mundo para que el enunciado sea verdadero, dados los hechos relacionados con el significado y la referencia. Pero el lenguaje, en virtud de su propia naturaleza, genera oraciones cuyo significado no encaja en esa imagen general. Éste es el caso de los enunciados de identidad con nombres e indéxicos. Dichos enunciados nunca son usados para transmitir su contenido referencial, el cual siempre es necesariamente verdadero o necesariamente falso (Perry 2001, pp. 119-120). 
Por lo general, a través de nuestras proferencias de enunciados singulares pretendemos que el interlocutor acceda al contenido referencial asociado a dicha proferencia; pretendemos que el interlocutor aprehenda la proposición singular expresada por la proferencia. Esto parece más claro cuando nuestro objetivo es construir una teoría, es decir, en un contexto teórico. Si yo digo que Venus es más grande que Marte, pretendo que el interlocutor aprehenda que Venus es más grande que Marte a pesar de que yo quizá tenga in mente la descripción el lucero vespertino asociada al nombre "Venus". ${ }^{22}$ Puede ser que, un mes más tarde, yo asocie con "Venus" la descripción el lucero matutino. A pesar de ello, por tratarse de un contexto teórico, mi objetivo principal es transmitir el contenido referencial u oficial de que Venus es más grande que Marte y no la información de que el lucero matutino es más grande que Marte. En un contexto teórico, por decirlo así, el contenido referencial es lo que cuenta, independientemente de las descripciones que se asocien con, por ejemplo, "Venus". El contenido referencial es el contenido semántico asociado con una proferencia, aunque en ocasiones tal vez no sea el objeto de la intención primaria del proferente. El contenido referencial es constante, no se ve afectado por la variabilidad, por ejemplo, del conocimiento descriptivo acerca de un objeto que sea el referente de un nombre propio. Es posible incluso que el proferente no sepa qué es lo que (semánticamente) está expresando. ${ }^{23}$

Como Perry señala, lo llamativo de las proferencias de identidad es que su contenido oficial tiene el estatus, digámoslo ahora así, de verdad analítica. ${ }^{24}$ ¿Qué función tienen en nuestras teorías las proferencias cuyo contenido oficial es analíticamente verdadero? Supongamos que estamos hablando de planetas y que formamos una base de creencias acerca de los mismos. ¿Qué función tiene una proferencia de un enunciado como "Héspero es Fósforo", cuyo contenido oficial es la verdad analítica que afirma que un objeto (Venus) es idéntico a sí mismo? Es innegable que proferencias como la citada forman parte de nuestras teorías.

En general, mediante una proferencia se pretende que el oyente acceda a su contenido oficial. Sin embargo, el quehacer de una proferencia cuyo contenido oficial es trivial no es, excepcionalmente,

${ }^{22}$ A partir de ahora, y para simplificar la exposición, me limitaré a enunciados singulares construidos con nombres propios.

${ }^{23}$ Esta idea básica del contenido semántico se encuentra desarrollada, por ejemplo, en Soames 2002.

${ }^{24}$ Para abreviar, en ocasiones utilizaremos la expresión "proferencia de identidad". 
transmitir su contenido oficial, aunque también lo hace a pesar de su trivialidad, sino impactar epistémica o cognoscitivamente a través de sus contenidos no oficiales. Este tipo de impacto difiere del impacto epistémico producido por proferencias que no expresan proposiciones triviales. En el caso de una proferencia de "Héspero es Fósforo" es evidente que su contenido oficial es que Venus es idéntico a sí mismo, pero también vehicula contenidos no oficiales como: el planeta asociado con "Héspero" a través de la convención que está siendo considerada al proferir "Héspero" es el mismo que el planeta asociado con "Fósforo" a través de la convención que está siendo considerada al proferir "Fósforo". Cabe afirmar, como Perry señala, que en general y por defecto las proferencias están dirigidas a transmitir un contenido oficial, pero en ocasiones, precisamente cuando el contenido oficial es trivial, el quehacer de las proferencias se enfoca hacia los contenidos no oficiales. Esta propuesta acomoda la idea intuitiva subyacente a la teoría (falsa) que reivindica que las proferencias de identidad expresan (semánticamente) una relación entre nombres, sin abandonar la idea de que una relación (reflexiva y sujeta a la ley de Leibniz) forma parte del contenido oficial de una proferencia de identidad.

Quizá el contenido referencial sea el candidato por defecto para ser lo que es dicho, pero en algunas circunstancias otros niveles del contenido pasan a ser -así lo diría yo- el asunto que nos ocupa. En los casos en los que el contenido referencial es trivialmente y necesariamente verdadero o falso, el contenido por defecto es sobrepasado. Los enunciados de identidad activan los mecanismos para reemplazar el contenido por defecto. (Perry 2001, pp. 121-122)

En Arrieta 2001 justifiqué una distinción entre la descripción y la especificación de un dominio. Mi objetivo ahora es volver a dicha distinción y darle acomodo en las distinciones propuestas por Perry. La posibilidad misma de que esta acomodación sea factible muestra que hay una sintonía clara entre dos concepciones acerca de la identidad que se han desarrollado por caminos relativamente distintos.

Supongamos que estamos desarrollando una teoría. Entiendo por teoría un conjunto, con las credenciales debidas, de enunciados o, más precisamente, de proferencias de los mismos. Una teoría es en todo caso una teoría sobre un dominio; por ejemplo, una teoría sobre planetas o sobre el vecindario del portal número 12 de la calle Santa Clara en San Sebastián. La teoría en cuestión proporciona 
información sobre dicho dominio. La idea básica es que esa información formulada en el lenguaje-objeto puede distribuirse en dos categorías. Por un lado hay proferencias que especifican el dominio mismo del que se ocupa la teoría y, por otro lado, hay proferencias que lo describen. En general, el dominio lo especificamos mediante algún término sortal como "planeta" o "vecinos de la calle c", pero, de hecho, en el desarrollo de la teoría esa especificación se detalla en un proceso diacrónico que va de la mano del enriquecimiento de la descripción del dominio. Lo interesante es que todas esas proferencias pertenecen al lenguaje-objeto. Con esto quiero señalar que tanto las proferencias especificadoras (al menos aquellas que nos interesan en este trabajo) como las descriptoras, en caso de ser formuladas en un lenguaje formal, se representarían como fórmulas de primer orden. La hipótesis es que las proferencias especificadoras, dentro del proceso de continua revisión y enriquecimiento de la teoría, constituyen presuposiciones con respecto a las proferencias descriptoras. ${ }^{25}$ Eso hace que haya una relación asimétrica entre proferencias especificadoras y descriptoras. Una revisión de creencias que afecte a las proferencias especificadoras trae consigo de forma derivada una revisión que afecta a las proferencias descriptoras. Por el contrario, las revisiones que afectan a la descripción del dominio no afectan a su especificación. En este sentido, es posible afirmar que las proferencias descriptoras presuponen las proferencias especificadoras.

Pasemos a un ejemplo. Si la teoría es sobre planetas, proferencias del enunciado "eso es un planeta" especifican el dominio. También lo hace una proferencia de "a eso que es un planeta lo voy a de-

${ }^{25} \mathrm{Al}$ afirmar que las proferencias de identidad (o de diferencia) se presuponen, quiero indicar el siguiente hecho. La información almacenada en la teoría puede resultar compatible tanto con la identidad como con la no identidad de $a$ y $b$. El problema es que, por distintas razones, aceptamos en la teoría que (supongamos) $a$ no es idéntico a $b$. Los planetas que vemos al amanecer y al atardecer pueden ser el mismo planeta (puede es una modalidad epistémica, ya que la identidad de ambos planetas es compatible con lo que sabemos), pero nosotros consideramos que Héspero no es Fósforo (aplicando, por ejemplo, el principio de la unicidad del nombre: dos nombres propios distintos se refieren a individuos distintos). Nosotros realizamos inferencias a partir de la no identidad. El problema es que, al descubrir que Héspero es Fósforo, todas estas inferencias quedan bloqueadas. La teoría es revisada en virtud de que se asumió erróneamente la no identidad de Héspero y Fósforo. En cualquier caso, recurrimos a la expresión de identidad para formular esas presuposiciones. Estoy pensando en asunciones como la del mundo cerrado (todo objeto del dominio tiene nombre) o de la unicidad del nombre. En este sentido, que afecta al carácter no monótono (o no monotónico) de las teorías, las proferencias de identidad se presuponen. Véanse cuestiones relacionadas con estrategias y lógicas no monótonas en Arrieta 1995 (en particular véase la p. 195, n. 8). 
nominar "Fósforo" o una proferencia de "a eso, supuestamente otro, que también es un planeta lo voy a denominar "Héspero" " o una proferencia de "Héspero es un planeta". Algunas de esas proferencias especificadoras se formulan en el metalenguaje, pero no así las que nos interesan. Una proferencia de "Héspero no es Fósforo" también es especificadora. Proferencias de "Fósforo aparece al amanecer", "Héspero aparece al atardecer", "Fósforo es más grande que el planeta Tierra", "en Fósforo no hay vida", "en Héspero no hay vida",... son descriptoras. Parece claro que una proferencia de "Héspero es Fósforo" tendría un impacto sobre la especificación del dominio y, de forma derivada, sobre su descripción: todas las propiedades de Fósforo pasarían a ser propiedades de Héspero. En cierta medida el descubrimiento de la identidad de Héspero y Fósforo rompe con ciertas presuposiciones asumidas cuando describimos el dominio de los planetas.

Resulta plausible pensar que las proferencias de existencia ("Neptuno existe", "Vulcano no existe") tienen el mismo estatus que las proferencias de identidad, aunque con sus características específicas. Lo que tienen en común es que todas ellas nos obligan a revisar la descripción del dominio de los planetas. La razón principal es que en esa descripción se presuponía, por ejemplo, la existencia de Vulcano. El impacto de este descubrimiento de no existencia afecta de forma directa a la especificación del dominio de los planetas, ya que tenemos que eliminar un elemento de dicho dominio: el dominio ha pasado de tener $m$ elementos a tener $m-1$. (Tendríamos que hablar de "elemento intencional". Este aspecto intencional hace que las proferencias de no existencia requieran un estudio específico, a pesar de tener características comunes con las proferencias de identidad.)

Consecuencias análogas se extraen del descubrimiento de que Héspero y Fósforo son el mismo planeta; también se produce una contracción de nuestra especificación de dicho dominio. No estoy en disposición de hacer una caracterización precisa de la frontera existente entre la especificación y la descripción de un dominio, pero sí me gustaría resaltar dos aspectos. Esa idea de presuposición parece ser clave para la identificación de una proferencia como especificadora o como descriptora. Por otro lado, parece revelador que entre las proferencias especificadoras se encuentren las de identidad y de existencia, es decir, aquellas que han dado quebraderos de cabeza a los filósofos y a los lógicos, y para las que se han propuesto análisis específicos, como aquellos que afirman que la existencia no es una propiedad o que la identidad no es una relación (y de serlo lo sería entre nombres). La imagen que acabo de proponer situaría a 
ambos tipos de proferencias entre las pertenecientes a la teoría; en particular, las proferencias de identidad se formulan en el mismo nivel en el que otras proferencias pertenecientes a la red teórica se formulan. De ahí que podamos hacer inferencias combinando sin ningún tipo de problema proferencias descriptoras con proferencias de identidad: de $P a$ y $a=b$, derivamos $P b$. Esto quiere decir que " $a$ " está por lo mismo tanto en "Pa" como en " $a=b$ ". Si una proferencia de " $a=b$ " fuera sobre nombres, esa inferencia debería entenderse de forma poco natural. Por otro lado, las proferencias de identidad (y de existencia) en lo que toca al contenido referencial son, valga la expresión, triviales. Esto las diferencia de las proferencias descriptoras. Esta inocencia referencial no evita que las proferencias de identidad tengan un impacto epistémico importante que afecta a la especificación del dominio.

Este modelo que se ha propuesto encaja con un análisis semántico como el que Perry ha propuesto: las proferencias de identidad tienen un contenido referencial, aunque trivial, y esto permite que se combinen sin traumas con otras proferencias, pero su quehacer fundamental o primario es el de afectar, vía el contenido no referencial, sobre toda esa capacidad epistémica concentrada en las creencias especificadoras del dominio que es objeto de estudio por parte de la teoría.

\section{Conclusiones}

Una proferencia de identidad verdadera expresa, pues, una relación (trivial o lógica o analítica) entre objetos. A pesar de ello, dicha proferencia no debe analizarse semánticamente de una forma diferente y ad hoc, como pretenden aquellos que defienden que la identidad es correferencialidad. Los hechos apuntan a que las proferencias de identidad conviven en nuestras teorías en igualdad de condiciones con proferencias ricas en contenido referencial. Además, no pueden eliminarse en favor de otras proferencias, ni puede prescindirse de ellas. En cualquier caso, el contenido referencial no es el único contenido que está ligado a una proferencia, aunque sí sea aquello hacia lo que la proferencia apunta en los contextos teóricos. En el caso de las proferencias de identidad, lo relevante no es el contenido referencial, sino el contenido reflexivo no referencial. El marco general es el mismo para todas las proferencias, pero, dada la analiticidad del contenido referencial de las proferencias de identidad, lo relevante pasa a ser el contenido no referencial, y se provoca un impacto epistémico sobre la especificación del dominio que es objeto de estudio. 
Esta característica no es exclusiva de las proferencias de identidad. Las proferencias de no existencia, aunque con sus características específicas, también nos llevan a la misma situación. Una hipótesis en la que hay que trabajar es si este esquema de tratamiento puede extenderse a otras proferencias especificadoras.

En cierta medida, las proferencias de identidad sí son diferentes, como los correferencialistas intuyen correctamente. En este marco quedan recogidas las razones, que las hay, a favor de la interpretación (falsa) de la identidad como correferencialidad. Las proferencias de identidad son imprescindibles e ineliminables en la tarea de especificación de un dominio y, a su vez, se insertan en nuestras teorías como cualquier otra proferencia. Esta característica, según el criterio ontológico que se ha propuesto (sección 2), hace de la identidad una relación; sin embargo, teniendo en cuenta que el criterio de la eficacia causal discrimina las relaciones reales de las que no lo son, sólo nos queda aceptar la identidad como relación de razón. ${ }^{26}$

\section{BIBLIOGRAFÍA}

Aristóteles, 1982, Tratados de Lógica, vol. 1. Tópicos, trad. Miguel Candel, Gredos, Madrid.

Armstrong, D.M., 1997, A World of State of Affairs, Cambridge University Press, Cambridge.

— 1978, A Theory of Universals, vol. 2. Universals and Scientific Realism, Cambridge University Press, Cambridge.

Arrieta, A., 2001, "Existencia e identidad: especificación frente a descripción de un dominio", Teorema, vol. 20, no. 3, pp. 5-19.

__ 1999, "Relatividad ontológica e identidad relativa y absoluta", en J.L. Falguera, U. Rivas y J.M. Sagüillo (comps.), La filosofía analítica en el cambio de milenio, Servicio Editorial, Santiago de Compostela, pp. 29-39.

, 1995, "Comentarios sobre la relación entre la programación lógica y las lógicas no monótonas", Teoría, vol. 10, no. 24, pp. 181-198.

Brower, J., 2001, "Medieval Theories of Relations", http://plato.stanford. edu/entries/relations-medieval/

${ }^{26}$ Quiero agradecer a Fernando Migura y a Manuel Pérez Otero sus correcciones, comentarios y críticas. Este trabajo se ha realizado en el marco del proyecto 1/UPV 00003.230-H-14780/2002. En el IV Coloquio Compostelano de Lógica y Filosofía Analítica presenté algunas partes de este trabajo. Los comentarios y observaciones de la audiencia (J.L. Falguera, C. Martínez, L. Villegas, J.M. Sagüillo, U. Rivas, A.R. Pérez Ransanz, entre otros) han contribuido a mejorarlo. Mi agradecimiento a un árbitro anónimo por sus observaciones, correcciones y críticas a las versiones previas de este trabajo. 
Frege G., 1996, Escritos filosóficos, trad. C. Ulises Moulines, Crítica, Barcelona.

—_, 1892, "Sobre sentido y referencia", en Frege 1996, pp. 172-198.

—_, 1891, "Función y concepto", en Frege 1996, pp. 147-172.

- 1879, Conceptografía, trad. H. Padilla, Instituto de Investigaciones Filosóficas-Unam, México (1972).

Gallois, A., 1998, Occasions of Identity, Clarendon Press, Oxford.

García Carpintero, M., 1996, Las palabras, las ideas y las cosas, Ariel, Barcelona.

Kripke, S., 1980, Naming and Necessity, Harvard University Press, Cambridge, Mass. (Versión en castellano: El nombrar y la necesidad, 2a. ed., trad. Margarita M. Valdés, Instituto de Investigaciones Filosóficas-UNAM, México, 1995.)

— 1979, “A Puzzle about Belief”, en A. Margalit (comp.), Meaning and Use, Reidel, Dordrecht, pp. 239-283.

— , 1971, "Identity and Necessity", en M.K. Munitz (comp.), Identity and Individuation, New York University Press, Nueva York, pp. 135164.

McGinn, C., 2001, Logical Properties, Oxford University Press, Oxford.

Noonan, H.W., 2001, Frege. A Critical Introduction, Polity Press, Cambridge.

Perry J., 2001, Reference and Reflexivity, CSLI, Stanford.

_ , 1977, "Frege on Demonstratives", Philosophical Review, vol. 86, no. 4, pp. 474-497.

Quine, W.V.O., 1970, Philosophy of Logic, Harvard University Press, Cambridge, Mass.

— 1960, Word and Object, MIT, Cambridge, Mass.

— 1953, From a Logical Point of View, Harvard University Press, Cambridge, Mass.

—, 1948, "On What There Is", en Quine 1953, pp. 1-20.

Soames, S., 2002, Beyond Rigidity: The Unfinished Semantic Agenda of Naming and Necessity, Oxford University Press, Oxford.

Wiggins, D., 2002, "Identity and Supervenience", en A. Bottani, M. Carrara y P. Giaretta (comps.), Individuals, Essence and Identity, Kluwer, Dordrecht, pp. 247-267.

Wittgenstein, L., 1921, Tractatus Logico-Philosophicus, trad. Luis M. Valdés, Tecnos, Madrid (2002).

Recibido el 11 de noviembre de 2003; revisado el 5 de noviembre de 2004; aceptado el 19 de enero de 2005. 